\title{
Generation of a high-quality electron beam for application in cyclotron autoresonance maser experiments
}

\author{
I. P. Spassovsky, ${ }^{1,2}$ G. P. Gallerano, ${ }^{1}$ J. J. Barroso,${ }^{3}$ and R. A. Corrêa ${ }^{3,4}$ \\ ${ }^{1}$ Dipartimento Sviluppo Tecnologie di Punta, Ente per le Nuove Tecnologie e l'Ambiente, P.O. Box 65-00044, Frascati, Italy \\ ${ }^{2}$ Sofia University, Department of General Physics, 5 A. Ivanov, Sofia 1126, Bulgaria \\ ${ }^{3}$ Instituto Nacional de Pesquisas Espaciais, 12201-970, São José dos Campos, São Paulo, Brazil \\ ${ }^{4}$ Institute for Plasma Research, University of Maryland, College Park, Maryland 20742
}

(Received 5 July 1995)

\begin{abstract}
In this paper we report on the numerical calculations and experimental testing of a nonadiabatically electrostatic pumped beam. These initial investigations are intended to lay the groundwork for generating a high-quality electron beam, which will be used for cyclotron autoresonance maser driving. The present gun geometry produces a beam at a voltage of $500 \mathrm{kV}$ in a $30-\mathrm{ns}$ pulse, with total power between 150 and $300 \mathrm{MW}$ The experimental data characterizing the beam properties are compared with numerical simulation results, indicating that the gun has operated essentially as predicted by theory.
\end{abstract}

PACS number(s): 41.75.Fr, 84.40.Ik

\section{INTRODUCTION}

Cyclotron autoresonance masers (CARM's) have some advantages over free-electron lasers (FEL's) as a CARM requires lower particle energies than a FEL for operating in the millimeter and submillimeter wavelength region. Moreover, the theoretical predictions for CARM's hold promise for high-power generation with high efficiency, of order $20-40 \%$ [1-3]. Unfortunately, there have been very few experimental demonstrations of such highpower handling capabilities [4-6], comparing other devices such as gyrotrons, traveling-wave tubes, and backward-wave tubes.

The most important and often most difficult task in CARM and FEL experiments is the generation and transport of a high-quality electron beam. A variation in velocity and energy can hinder the electron bunching process and sharply decreases the growth rate and efficiency. For example, the CARM operates well when the pitch angle spread $\delta \varphi$ over the interaction length $L$ is smaller than $2 \pi$. This limitation can be expressed by the axial velocity and the energy spreads as $\delta v_{\|} / v_{\|} \leq 2 \pi / k_{\|} L$ and $\delta \gamma / \gamma \leq 1 / N$, where $v_{\|}$and $k_{\|}$are the electron velocity and radiation wave number in the axial direction, $N$ is the number of cyclotron turns in the interaction region, and $\gamma$ is the relativistic mass factor. Electron energy and velocity spreads can be produced by the beam emittance, electric or magnetic field gradients in the pumping section, any voltage variation during the acceleration, as well as by the beam space charge. Usually the pumping section (where the electrons are set in rotational motion) is a configuration employing single or multiple nonadiabatically modulated electric or magnetic fields. The nonadiabatic changes produce strong off-axis fields, resulting in higher perpendicular velocity for the outer beam particles than for the inner ones [7]. At the same time the inner particles have higher axial velocity. Consequently, a major experimental requirement is to use an emitter as thin as possible. Another important parameter, characterizing the beam quality, is the emittance $\varepsilon=\beta \gamma \delta \varphi r_{b}$, where $\beta$ is the normalized electron velocity and $r_{b}$ is the beam radius. Even if the beam electrons are assumed to all have the same energy, the emittance produces a spread in axial energy and velocity. There are many important contributions to the beam emittance. Some of them are nonuniform cathode emission, cathode surface roughness, nonlinear accelerating electric field or focusing fields, nonlinear space-charge forces, magnetic field aberration or misalignment, and mismatching between focusing elements. Obviously, special attention has to be paid during the design and fabrication of the gun to keep the beam emittance as small as possible to ensure efficient operation at short wavelengths. Taking into account all of the above-mentioned particular features and characteristics of the beam, we propose in this paper a configuration of a high-power electron gun to generate a high-quality, several-hundred-ampere electron beam.

\section{EXPERIMENT, PROCEDURE, AND RESULTS}

A schematic diagram of the experimental setup is shown in Fig. 1. A double coaxial pulse line accelerator "PERUN-2" generates a 500-kV pulse with duration of 30 ns. The cathode is a hollow stainless steel short cylinder with external and internal diameters of 64 and $31 \mathrm{~mm}$, respectively. A stepped-iris anode closing around the cathode provides electric field modulation. The iris has an inner diameter of $48 \mathrm{~mm}$ and a thickness of 25 $\mathrm{mm}$. Moreover, moving it forward and backward we can also change the diode impedance, which is useful for beam current and voltage control. The diode operates in the field emission regime with the electrons being extracted from the annular graphite ring mounted on the cathode end surface. All diode elements have been fabricated with rounded edges to minimize unwanted electron emission. The emitter ring has a diameter of $45 \mathrm{~mm}$ and a width of $1.5 \mathrm{~mm}$. The reason for using such a configuration is twofold. First, the leading surface of the hollow ringlike cathode must be large enough to keep the electric field quite homogeneous over the region close to 


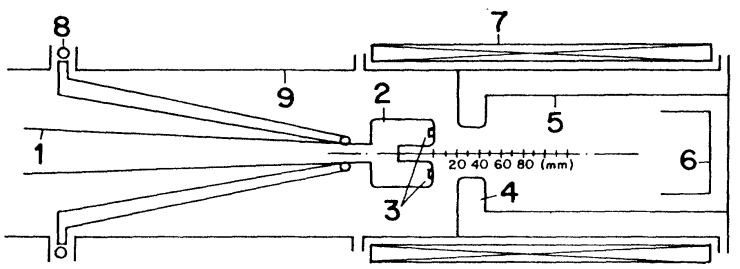

FIG. 1. Experimental setup: 1, matching cone; 2, cathode; 3, emitter ring; 4, stepped iris anode; 5 , drift tube; 6 , collector; 7 , solenoidal magnet; 8, Rogowsky coil; and 9, vacuum chamber.

the emitter; second, the emitter is positioned far from the side-on surface to eliminate any strong edge-current emission. Both the anode and cathode are immersed in a uniform magnetic field with a pulse duration of $12 \mathrm{~ms}$ provided by a solenoidal magnet. The homogeneous part of the axial magnetic field distribution extends over $12 \mathrm{~cm}$ from the emitter, which is sufficient for the beam formation. Downstream from the modulating aperture the beam propagates in a $62-\mathrm{mm}$-diam drift tube.

During the experimental test two different emitters were used. The first one has been machined to be $\sim 0.2$ $\mathrm{mm}$ bulging out from the cathode body while the second one was $\sim 0.3 \mathrm{~mm}$ above the metal surface. Certainly, both rings had rounded edges for the reason mentioned above. Slightly bulged emitters were used because when the emitting surface coincides with the cathode edge, the beam current is not high enough. Furthermore, a fringed emission from the canal housing the ring was observed.

The previously described arrangement was optimized by means of a ray-tracing code to model the beam trajectory [8]. The calculation was motivated in order to produce a laminar electron flow with low velocity spread. In the design study, the diode voltage, the beam current, and the pitch ratio $\alpha=v_{\perp} / v_{\|}$were kept constant, whereas both the magnetic field and the geometrical configuration were varied to minimize the velocity spread.

Figure 2 shows the parallel and perpendicular velocities versus axial position $z$ (beam current is $I_{b}=700 \mathrm{~A}$ ). The modulation aperture is positioned $21 \mathrm{~mm}$ from the emitter. As can be seen from the figure, the axial velocity reaches its full value around the entrance to the anode. However, the transverse velocity grows continuously when the beam passes through the stepped-iris aperture. From this result we can conclude that the diaphragm plays an important role in the beam pumping process, thus acting as a kicker. Since $v_{\|}$remains almost constant along the pumping section $(30 \mathrm{~mm} \leq z \leq 45 \mathrm{~mm})$ and only $v_{\perp}$ increases, this leads to an increase of the pitch ratio, whose dependence on the axial coordinate is also depicted in Fig. 2.

In general, a beam pumping process is quite complicated because there are many factors that can degrade the beam quality. Usually the problem related to the origin of the initial beam spread is one of the most important tasks to solve. This spread mainly arises from the nonuniform electric field close to the emitter. In practice, the calculations show that the transversal electric field near the cathode is likely to be quite nonuniform even though

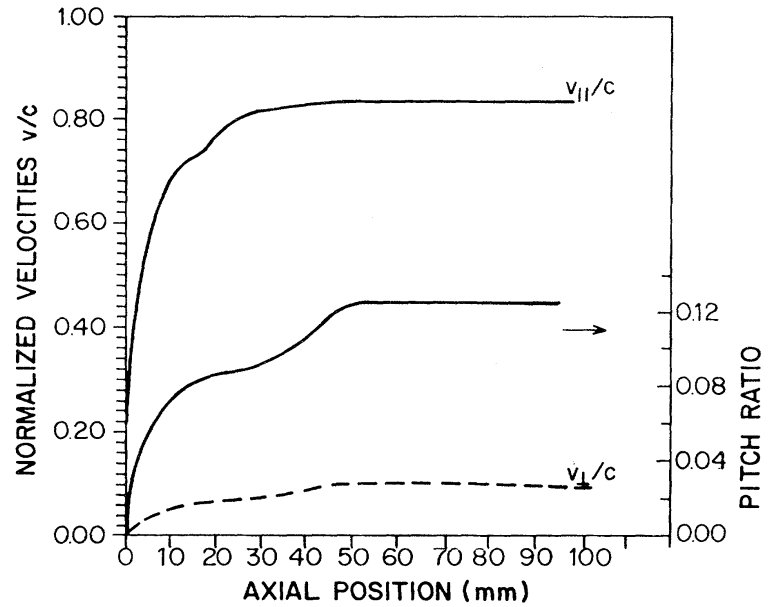

FIG. 2. Pitch ratio, normalized axial (solid line) and perpendicular (dashed line) velocities as functions of axial position.

a very narrow emitter is used. This is supported by the values of $E_{r}$ experienced by the outermost and innermost rays, which are calculated to be 17 and $7.5 \mathrm{kV} / \mathrm{cm}$, respectively, at the emitting surface. This difference causes a variation in the transverse velocity across the beam. For example, close to the cathode, the transverse velocity spread varies from $13 \%$ to $17 \%$ for a beam current varying in the 300-700 A range (Fig. 3). Downstream of the anode, $E_{r}$ becomes more homogeneous and the spread drastically goes down to $1 \%$. However, in the pumping section, the radial electric field is characterized by a continuously increasing dispersion, which increases the transverse velocity spread. Varying the beam current from 700 to $300 \mathrm{~A}$, the calculations indicate that the transverse velocity spread decreases from about $7 \%$ to

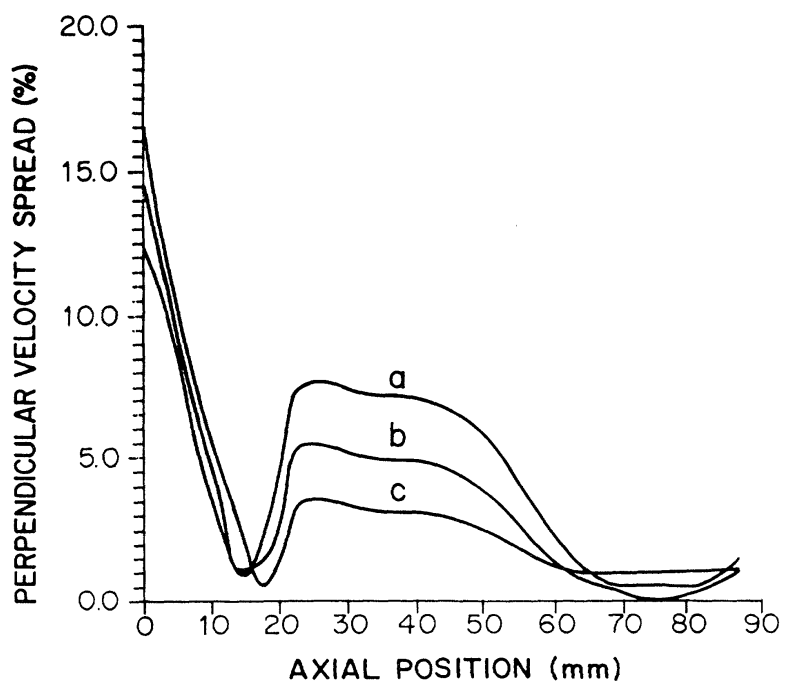

FIG. 3. Perpendicular velocity spread $\delta v_{\perp}$ as a function of axial position for different beam currents: (a) $300 \mathrm{~A}$, (b) $500 \mathrm{~A}$, and (c) $700 \mathrm{~A}$. 
$2 \%$. Beyond the stepped-iris diaphragm, the beam propagates with a maximum spread of $1.5 \%$ for a $300 \mathrm{~A}$ beam current and $3 \%$ for $700 \mathrm{~A}$. This is because space-charge effects are less significant as the magnetic field is constant along the drift tube. In contrast to $E_{r}$, the axial electric field near the emitting surface is much stronger, $\sim 200$ $\mathrm{kV} / \mathrm{cm}$, with a very small variation of about $3 \%$, which translates into a negligibly small parallel velocity spread (Fig. 4). The longitudinal electric field experienced by the innermost and outermost rays coincide throughout the region between cathode and anode. This has a beneficial effect on the gun performance as the axial velocity spread is sharply reduced before reaching the pumping section. At the beginning of the aperture, however, $E_{z}$ undergoes a nonuniform fluctuation across the beam radius, which contributes to an increasing velocity spread. At this place a nonadiabatic energy exchange does occur. Part of the energy belonging to the axial motion is transferred to the energy of the transversal motion, i.e., nonadiabatic pumping is achieved.

The electron gun was tested by changing the matching between the pulse-forming line and the diode in order to tune the beam current and voltage. An important part of any beam-forming experiment is the accurate measurement of the beam parameters. We have used several diagnostics on our beam line that have allowed us to determine the full diode and actual beam current, diode voltage, beam position and profile, velocity spread, and pitch ratio $\alpha$. The diode voltage has been measured by the voltage monitor, represented by a capacitance divider situated at the end of the coaxial line in an oil insulated region. Close to it at the beginning of the tapered section, matching the line with the diode, a Rogowsky coil has been mounted for full diode current reading. These two probes have been used to control also the shape of the pulses. The actual current has been measured using a

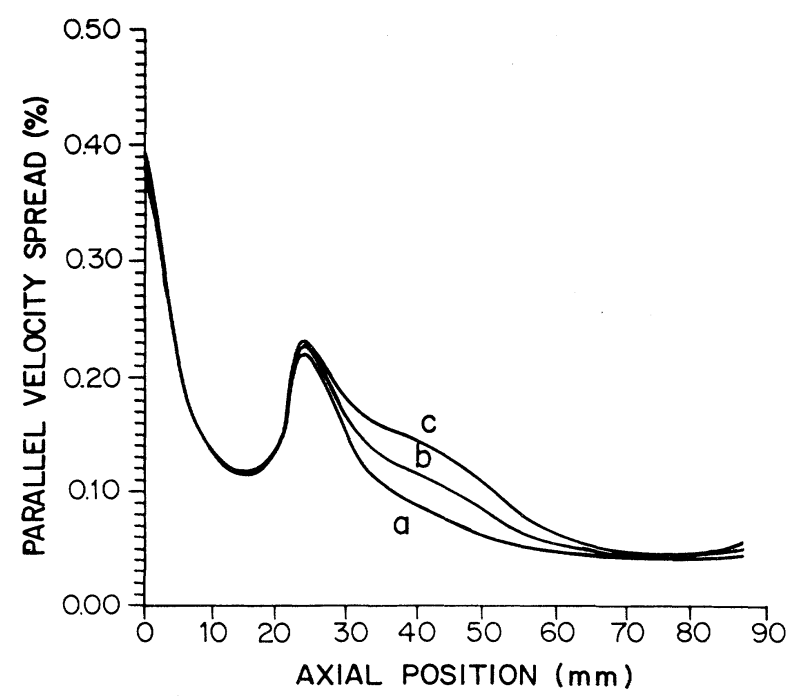

FIG. 4. Parallel velocity spread $\delta v_{\|}$as a function of axial position for different beam currents: (a) $300 \mathrm{~A}$, (b) $500 \mathrm{~A}$, and (c) $700 \mathrm{~A}$.
Faraday cup made from a graphite cylinder with internal tapering to better collect the beam electrons. The collector is placed in the electron beam path and is connected to a ground through a low resistance.

More difficulties have arisen when measuring the pitch ratio and velocity spread. The problem came about due to the relatively small $\alpha$ values and low velocity spread. For their evaluation we have used three diagnostics. First, using a diamagnetic loop placed on a plane of constant axial position and encircling the beam path we measured the pitch ratio. All the $\alpha$ values derived from the loop were over 1.5 times larger than those predicted by the computer program EGUN. Moreover, the discrepancy increases with increasing current. With the purpose of verifying the results from diamagnetic measurements, beam-trace pictures have been taken along the $z$ axis at regular steps of $1 \mathrm{~cm}$. The photographs have been compared with the beam profile and thickness calculated from the computer simulation. We have found that the experimental data closely resemble the computational predictions for an emitter ring bulging out by $0.2 \mathrm{~mm}$. This scan has been done for the magnetic field varying between 2.0 and $2.5 \mathrm{kG}$. Using the beam-tracing scan we analyzed and calculated the pitch ratio. However, in contrast to the results from the diamagnetic loop, in this case the best fit with the numerical computation has been observed at a magnetic field of $2.4 \mathrm{kG}$ and beam current $I_{b}=500 \mathrm{~A}$. Even for very clear beam profile pictures, the measured pitch ratio is $30-40 \%$ above the value calculated by EGUN. Unfortunately, it was impossible to take photographs in front of the stepped-iris aperture; otherwise the diode configuration would have to be changed. Instead of this, another test has been performed to evaluate the full beam current emitted from the cathode and propagating toward the anode. The measurements have been done by grounding the aperture and drift tube through the collector. Thus we were able to measure the full flowing current directed to the right-hand side of the cathode. The observed current in this case was about $15 \%$ higher than the collected one. This means that the diaphragm scrapes off the outer edge of the annular beam. At the same time the diode current was twice as high as the one measured with the collector. Obviously it happened because only the front part of the cathode cup

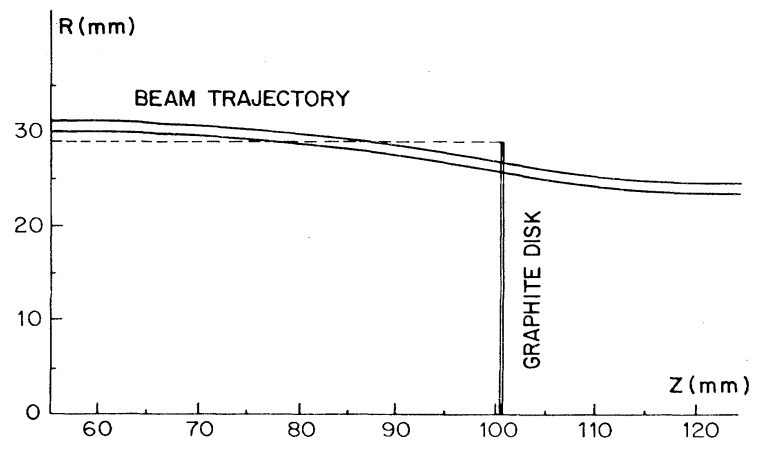

FIG. 5. Simple scheme of the experiment for the velocity spread measurement. 
is immersed in a homogeneous field. The rest of the cup is in a decreasing field that is two times smaller at the cathode shank. Finally, to evaluate the rotational velocity spread we used a diagnostic proposed by Bratman, Denisov and Samsonov [9]. Moving a thin graphite disk along the $z$ axis (Fig. 5), we measured the current collected on it at fixed beam parameters and magnetic field $\left(I_{b}=680 \mathrm{~A}\right.$ and $\left.B=2.4 \mathrm{kG}\right)$. A comparative analysis of the calculated trajectory and measurements indicates a transverse velocity spread of less than $4 \%$ (the calculated spread is $3 \%$ ).

\section{CONCLUSION}

The experimental test and simulation of a nonadiabatically pumped electron beam have shown that the proposed gun can be used successfully for generating a laminar electron flow with appropriate pitch ratio and low velocity spread. The measured data are consistent with the predictions of simulation, apart from a disagreement between measured and calculated values of the pitch ratio $\alpha$. Similar effect has been observed in the experiments carried out by Temkin and co-workers [10,11] and Calame et al. [12]. Both groups measured the velocity ratio with a capacitive probe. Temkin and co-workers attribute the poor agreement between the experiment and calculation of inadequate modeling of space-charge effects. In contrast, Calame et al. attribute the discrepancy to a self-axial magnetic field, which opposes the applied magnetic field. It results in a reduction of $B_{0}$ ( $B_{0}$ is the external field). Therefore, $\alpha \propto v_{\perp} \propto E_{\perp} / B_{0}$, where $E_{1}$ is the average transverse electric field in the diode region. Hence $\alpha$ increases proportionally to the reduction of $B_{0}$. In our case the perpendicular velocity is not very high and consequently the self-magnetic field cannot decrease drastically the guiding field. Moreover, the space charge is considerably high because the current is several hundred amperes. For this reason it is more realistic to attribute disagreements to space-charge effects. In addition, some errors could come from voltage fluctuation, difficulties in reading data from diamagnetic loop, and any misalignments.

Finally, we remark the sensitivity of the CARM efficiency to velocity spread. In a recent study [13] on the effect of transverse velocity on Doppler up-shifted operation at the fundamental and cyclotron harmonic operation for various types of gyrodevices, it has been demonstrated that CARM operation in gyrotwistrons has an advantage over second-harmonic gyrotwistrons if the scaled velocity spread $\left(\delta_{\perp} / v_{\perp}\right) \sqrt{10^{-2} / I_{0}}$ is below $\sim 0.15$, where $I_{0}$ denotes the normalized beam current. On considering the typical parameters of the Lawson et al. high-power gyroklystron experiment [14] in connection with the beam characteristics provided by our nonadiabatic gun, namely, $\delta v_{\perp} / v_{\perp} \sim 4 \%$ at a $680 \mathrm{~A}$ beam current, one finds that the scaled velocity spread is on the order of 0.1 . Therefore, this indicates that CARM operation is more efficient than cyclotron harmonic operation so as to increase the operating frequency of such a device at a fixed value of the external magnetic field.

\section{ACKNOWLEDGMENT}

One of the authors (I.P.S.) undertook part of this work with the support of the "ICTP Programme for Training and Research in Italian Laboratories, Trieste, Italy."
[1] V. L. Bratman, N. S. Ginzburg, G. S. Nusinovich, M. I. Petelin, and P. S. Strelkov, Int. J. Electron. 51, 541 (1981).

[2] K. D. Pendergast, B. D. Danly, R. J. Temkin, and J. S. Wurtele, IEEE Trans. Plasma Sci. 16, 122 (1988).

[3] C. Chen and J. S. Wurtele, Phys. Rev. Lett. 65, 3389 (1990).

[4] B. G. Danly, F. V. Hartemann, T. S. Chu, P. Legorburu, W. L. Menninger, R. J. Temkin, G. Failon, and G. Mourier, Phys. Fluids B 4, 2307 (1992).

[5] G. Bekefi, A. Di Rienzo, C. Leibovich, and B. G. Danly, Appl. Phys. Lett. 54, 1302 (1989).

[6] J. G. Wang, R. M. Gilgenbach, J. J. Choi, C. A. Outten, and T. A. Spencer, IEEE Trans. Plasma Sci. 17, 906 (1989).

[7] B. Piosczyk, Int. J. Electron. 67, 447 (1989).

[8] W. B. Herrmannsfeldt, Stanford University, SLAC Report No. 166, 1979 (unpublished).

[9] V. L. Bratman, G. G. Denisov, and S. V. Samsonov, in
Proceedings of the Second International Workshop on Strong Microwaves in Plasmas, edited by A. Litvak (Institute of Applied Physics, Nizhny Novgorod, Russia, 1993), Vol. 2, p. 690.

[10] W. Guss, T. Grimm, K. E. Kreischer, J. T. Polevoy, and R. J. Temkin, Appl. Phys. 69, 3789 (1991).

[11] S. Alberti, B. G. Danly, G. Gilotta, E. Giguet, T. Kimura, W. L. Menninger, J. L. Rullier, and R. J. Temkin, Phys. Rev. Lett. 71, 2018 (1993).

[12] K. P. Calame, J. Cheng, B. Hogan, W. Lawson, C. D. Striffler, P. L. Latham, and V. Orwin, IEEE Trans. Plasma Sci. 22, 476 (1994).

[13] G. S. Nusinovich, P. E. Latham, and H. Li, IEEE Trans. Plasma Sci. 22, 796 (1994).

[14] W. Lawson, J. P. Calame, B. Hogan, P. E. Latham, M. E. Read, V. L. Granatstein, M. Reiser, and C. D. Striffer, Phys. Rev. Lett. 67, 520 (1991). 